\title{
MicroRNA-99b predicts clinical outcome of osteosarcoma and suppresses tumor cell proliferation, migration and invasion
}

\author{
Xin Shi ${ }^{*}$ and Xingfa Guan ${ }^{\dagger}$
}

\begin{abstract}
Background: Osteosarcoma (OS) is a malignancy predominantly occurred in children and adolescents. Numerous microRNAs are involved in the pathogenesis of various cancers. This study aimed to investigate the expression profiles of miR-99b and its prognostic value in OS patients, and further analyze the biological function of miR-99b in the tumor progression by using OS cells.

Methods: Expression of miR-99b was measured using quantitative real-time PCR. Kaplan-Meier survival curves and Cox regression analysis were performed to evaluate the prognostic value of miR-99b. OS cell lines were used to investigate the effects of miR-99b on cell proliferation, migration and invasion.

Results: A significant decreased expression of miR-99b was observed in the OS tissues and cell lines respectively compared with the normal tissues and cells. Aberrant expression of miR-99b was associated with the patients' metastasis and TNM stage, and could be used to predict the prognosis of OS. The expression of miR-99b was regulated in vitro by cell transfection, and we found that the overexpression of miR-99b led to suppressed cell proliferation, migration and invasion, whereas the knockdown of miR-99b resulted in the opposite results.

Conclusions: In one word, the aberrantly expressed miR-99b serves a prognostic biomarker for OS patients. OS cell proliferation, migration and invasion can be inhibited by the overexpression of miR-99b, suggesting that the methods to increase miR-99b expression may be novel therapeutic strategies in OS.
\end{abstract}

Keywords: Osteosarcoma, miR-99b, Prognosis, Biological function, Tumor progression

\section{Introduction}

Osteosarcoma (OS) is a primary bone tumor and characterized by the bimodal distribution that mainly occurs in children and adolescents [1]. Statistics indicate that the incidence of OS is approximately 4.4 per 100,000, and this malignancy ranks the second common primary tumor in bone [2]. Some risk factors have been identified to be closely related with the occurrence of OS, including medical history of radiation or chemotherapy, benign bone lesions and some genetic conditions [3]. Surgical operation is the predominant chose for OS treatment, and the adjuvant therapy, such as chemotherapy, contributes to the reduction of mortality [4]. Currently, the

\footnotetext{
* Correspondence: shixin196905@163.com

${ }^{+}$Xin Shi and Xingfa Guan are the first two authors contributed equally to this work

Department of Orthopaedics, Qilu Hospital Huantai Branch, No.2198, Huantai Road, Zibo 256400, Shandong, China
}

5-year survival rate of this malignancy has reached to $80 \%$ [5]. However, the prognosis remains dismal for the OS patients diagnosed with advanced tumors [6]. Thus, accurate prognosis and efficient therapy are urgently needed to improve the treatment of OS.

Targeted cancer therapy has received increasing attention in the cancer research field as a large number of deregulated key molecules have been identified in various tumors [7, 8]. Numerous microRNAs (miRNAs) with abnormal expression patterns have been detected in human cancer samples, and they are considered to be involved in the pathogenesis of various cancers $[9,10]$. MiRNAs are a groups of small noncoding RNAs with the important regulatory function in the gene expression at post-transcriptional levels [11]. In addition, miRNAs serve critical roles in the regulation of some cell processes, such as cell proliferation, migration, invasion, 
differentiation and cell apoptosis [12]. In OS, some aberrant miRNAs have also been identified, such as miR-124 [13] and miR-491 [14], with close correlation with diagnosis, prognosis and tumorigenesis. Decreased expression of microRNA-99b (miR-99b) has been reported in several types of human cancer, including gastric cancer [15] and colorectal carcinoma [16]. The regulatory role of miR-99b in the chemotherapy of OS has been analyzed in a study by Gougelet et al. [17], but its expression pattern and clinical significance in OS remain elusive.

In this study, the expression of miR-99b in the patients with OS was investigated, and the potential clinical significance of miR-99b was evaluated. To further understand the functional role of miR-99b in OS progression, its effects on tumor cell processes were assessed.

\section{Materials and methods}

\section{Patients and tissue collection}

OS tissues and paired adjacent normal tissues were collected from 110 patients who had been subjected to resection surgery in Qilu Hospital Huantai Branch from June 2010 to May 2013. The patients included 59 males and 51 females with an average age of $20.98 \pm 5.08$ years old (age range of 9-29 years old). None of patients had received any therapy prior to the surgery, and all the patients were pathologically diagnosed as OS after the surgery. The collected tissues were graded into IA -IIA stage $(n=38)$ and IIB - III stage $(n=72)$ according to the Enneking - Musculoskeletal Tumour Staging System. All the tissues were frozen in liquid nitrogen and stored at $-80^{\circ} \mathrm{C}$ for further use. The demographic and clinicopathological characteristics and the survival information in a 5-year follow-up survey (range of 0-60 months) were recorded for the subsequent analyses. The cases died from other events were excluded from this study. A signed written informed consent was obtained from each patient and the experimental procedures were in accordance with the guideline of the Ethics Committee of Qilu Hospital Huantai Branch.

\section{Cell culture}

Four OS cell lines (HOS, MG63, SaOS2 and U2OS) and one normal human osteoblast cell line (hFOB1.19) were obtained from the Shanghai Cell Bank of Chinese Academy of Science (Shanghai, China). The cells were maintained in DMEM medium (Gibco, CA, USA) supplemented with $10 \%$ FBS (Gibco, CA, USA) and cultured at $37^{\circ} \mathrm{C}$ with $5 \% \mathrm{CO}_{2}$.

\section{Cell transfection}

To regulate the expression of miR-99b in OS cells, miR-99b mimic (5'-CACCCGUAGAACCGACCUUGCG-3'), miR99b inhibitor (5'-CGCAAGGUCGGUUCUACGGGUG-3') and the negative control sequence (miR-NC, $5^{\prime}$-UUCUCC GAACGUGUCACGUTT-3') were synthesized in Ribobio (Guangzhou, China). The vectors above were separately transfected into the OS cells using Lipofectamine 2000 (Invitrogen, Carlsbad, CA, USA) following the manufacturers' protocols.

\section{RNA extraction and quantitative real-time polymerase chain reaction (qRT-PCR)}

Total RNA was extracted from the tissues and cells using the TRIzol reagent (Invitrogen, Carlsbad, CA, USA) as per the standard method. The synthesis of cDNA from the RNA was performed using a PrimeScript RT reagent kit (TaKaRa, Shiga, Japan) as per the manufacturers' instruction. The expression of miR-99b was measured by qRT-PCR, which was carried out using a SYBR green I Master Mix kit (Invitrogen, Carlsbad, CA, USA) on a 7500 Real-Time PCR System (Applied Biosystems, USA). U6 was used as an endogenous control, and the final expression value was calculated using the $2^{-\Delta \Delta \mathrm{Ct}}$ method. The primers used for this analysis were as follows: miR-99b F: 5'-GCCGAGCACCCGTAGAACCG-3', R: 5'-CTCAACTGGTGTCGTGGA-3'; U6 F: 5' CTCGCTTCGGCAGCACA-3', R: 5' -AACGCTTCAC GAATTTGCGT-3'.

\section{Cell proliferation analysis}

After $48 \mathrm{~h}$ of cell transfection, the tumor cells were seeded in 96-well plates to examine the ability of proliferation using the MTT method. The cell plates were maintained in an incubator at $37{ }^{\circ} \mathrm{C}$ for 3 days, and $10 \mu \mathrm{L}$ MTT $(5 \mathrm{mg} / \mathrm{mL})$ was added at the time points of $0,24,48$ and $72 \mathrm{~h}$ followed by further $4 \mathrm{~h}$ incubation. Then a volume of $150 \mu \mathrm{L}$ DMSO was added in the wells. After the incubation, a microplate reader was adopted to measure the absorbance of the cells at $490 \mathrm{~nm}$.

\section{Cell migration and invasion analysis}

Transwell chambers (Corning, USA) with Matrigel precoating (for invasion assay) or without Matrigel coating (for migration assay) were used to analyze the migration and invasion abilities of the OS cells. Tumor cells with serum-free culture medium were seeded into the upper chambers, and the lower chambers included medium contained 10\% FBS. After $48 \mathrm{~h}$ incubation at $37^{\circ} \mathrm{C}$, the cells in the lower chambers were stained and the cell number in five random fields was counted under an inverted microscope (Olympus Corporation, Tokyo, Japan).

\section{Statistical analysis}

SPSS 21.0 software (IBM, Chicago, IL) and GraphPad Prism 7.0 software (GraphPad Software, Inc., USA) were used for data analyses. All the experiments and examinations were performed at least three times. Data were 
expressed as mean \pm SD. Student's $t$ test and one-way ANOVA were used to assess the between-group differences. Relationship between miR-99b and clinical features was analyzed using Chi-square test. The Kaplan-Meier (KM) method was used to plot survival curves for the OS patients, and Cox regression analysis was applied to evaluate the prognostic value of miR-99b. $P<0.05$ was considered statistically significant.

\section{Results}

Decreased expression of miR-99b in OS

According to the qRT-PCR, we found that the expression of miR-99b in the tumor tissues was obviously downregulated compared with the normal tissues $(1.34 \pm 0.80$ vs. $2.91 \pm 1.09, P<0.001$, Fig. 1a). In addition, the expression patterns of miR-99b were further confirmed in the OS cells, which also performed a significantly lower expression than the normal cells (all $P<0.01$, Fig. 1b).

\section{Association between miR-99b and the clinicopathological characteristics of the patients}

All the demographic and clinical features were summarized in Table 1. By using the median value (1.245) of miR-99b expression, the OS patients were grouped into low $(n=56)$ and high $(n=54)$ miR-99b expression groups. From the data of Chi-square test, we observed that the expression of miR-99b was associated with metastasis $(P=0.008)$ and TNM stage $(P=0.003)$. However, no relationship was found between miR-99b and age, gender, tumor size or differentiation (all $P>0.05$ ).

\section{Prognostic significance of miR-99b in the patients with}

\section{OS}

Given the dysregulation in the expression of miR-99b in OS clinical samples, this study further investigated the
Table 1 Association of miR-99b and the clinical characteristics of the OS patients

\begin{tabular}{|c|c|c|c|c|}
\hline \multirow[t]{2}{*}{ Features } & \multirow{2}{*}{$\begin{array}{l}\text { Total No. } \\
N=110\end{array}$} & \multicolumn{2}{|c|}{ miR-99b expression } & \multirow{2}{*}{$P$ values } \\
\hline & & Low $(n=56)$ & High $(n=54)$ & \\
\hline Age (Years) & & & & 0.724 \\
\hline$\leq 20$ & 45 & 22 & 23 & \\
\hline$>20$ & 65 & 34 & 31 & \\
\hline Gender & & & & 0.989 \\
\hline Female & 51 & 26 & 25 & \\
\hline Male & 59 & 30 & 29 & \\
\hline Tumor size (cm) & & & & 0.311 \\
\hline$\leq 8$ & 66 & 31 & 35 & \\
\hline$>8$ & 44 & 25 & 19 & \\
\hline Distant metastasis & & & & 0.008 \\
\hline Negative & 55 & 21 & 34 & \\
\hline Positive & 55 & 35 & 20 & \\
\hline Differentiation & & & & 0.876 \\
\hline Well/moderate & 67 & 34 & 33 & \\
\hline Poor & 43 & 22 & 21 & \\
\hline Clinical stage & & & & 0.003 \\
\hline $\mid A-\| A$ & 38 & 12 & 26 & \\
\hline$\|B-I\|$ & 72 & 44 & 28 & \\
\hline
\end{tabular}

clinical significance of miR-99b in the prognosis of OS. As shown in Fig. 2, the patients with low levels of miR99b had markedly shorter survival time compared with those with high levels of miR-99b (log-rank $P=0.015)$. According to the 60 months follow-up survey, the OS patients had a median survival time of 48 months, and this value in the cases with low miR-99b was 42 months. To confirm whether the relationship between miR-99b

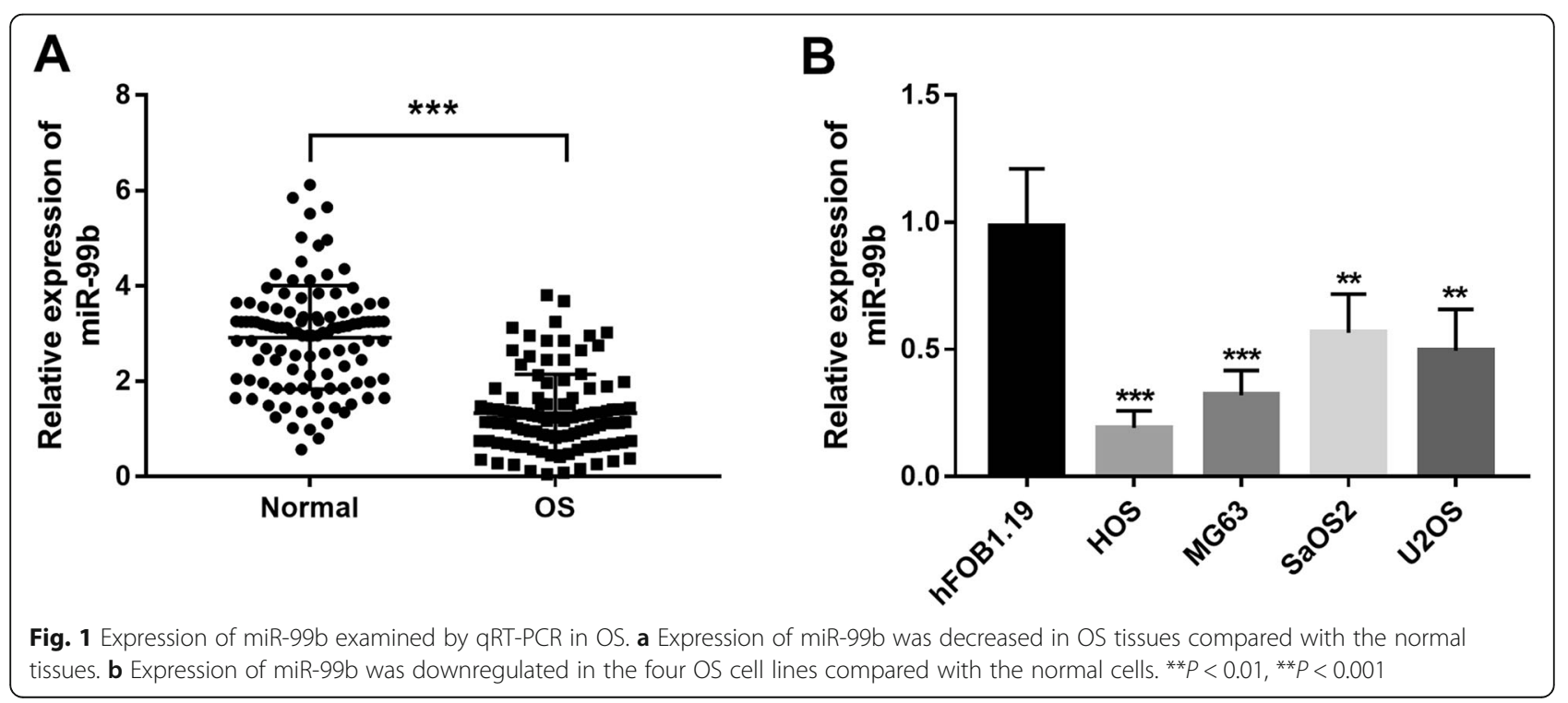




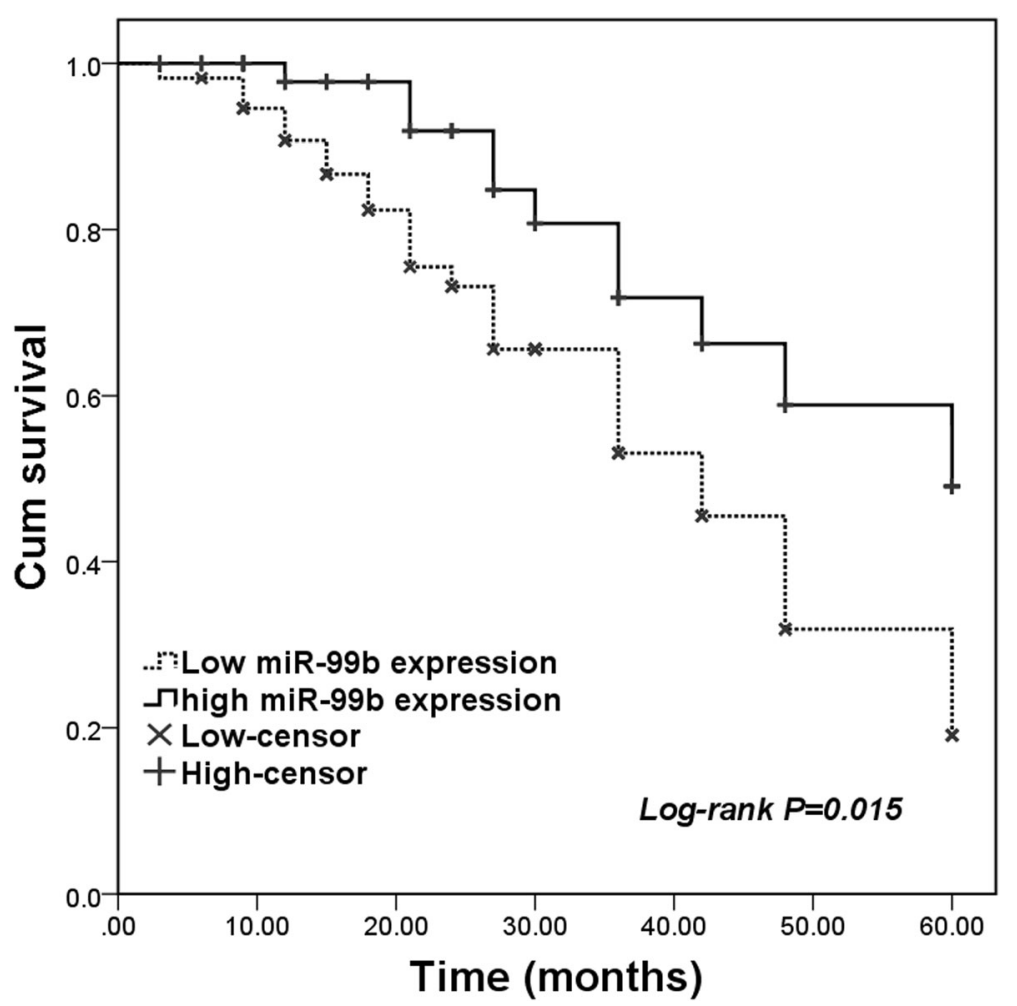

Fig. 2 Kaplan-Meier survival curves in the patients with OS. Patients with low expression of miR-99b had shorter survival time than those with high miR-99b expression. Log-rank $P=0.015$

and the overall survival was independent, a Cox regression analysis was performed. The univariate analysis results revealed that the expression of miR-99b, distant metastasis, differentiation and clinical stage were all associated with the patients' overall survival (all $P<0.05$,
Table 2). The further multiple assay data indicated that miR-99b expression was an independent prognostic indicator for the overall survival in the patients with $\mathrm{OS}(\mathrm{HR}=2.897,95 \% \mathrm{CI}=1.231-4.628$, $P=0.019$ ).

Table 2 Cox regression analysis in the patients with OS

\begin{tabular}{|c|c|c|c|c|c|c|}
\hline \multirow[t]{2}{*}{ Variables } & \multicolumn{3}{|c|}{ Univariate analysis } & \multicolumn{3}{|c|}{ Multivariate analysis } \\
\hline & $\mathrm{HR}$ & $95 \% \mathrm{Cl}$ & $P$ value & $\mathrm{HR}$ & $95 \% \mathrm{Cl}$ & $P$ value \\
\hline miR-99b & 3.012 & $1.622-6.185$ & 0.009 & 2.897 & $1.231-4.628$ & 0.019 \\
\hline \multicolumn{7}{|l|}{ Low vs. High } \\
\hline Age (years) & 0.867 & $0.456-1.649$ & 0.664 & 0.937 & $0.482-1.822$ & 0.848 \\
\hline \multicolumn{7}{|l|}{$>20$ vs. $\leq 20$} \\
\hline Gender & 1.258 & $0.650-2.435$ & 0.496 & 1.117 & $0.552-2.258$ & 0.758 \\
\hline \multicolumn{7}{|l|}{ Male vs. Female } \\
\hline Tumor size $(\mathrm{cm})$ & 0.940 & $0.490-1.803$ & 0.853 & 1.022 & $0.517-2.020$ & 0.950 \\
\hline \multicolumn{7}{|l|}{$>8$ vs. $\leq 8$} \\
\hline Distant metastasis & 2.018 & $1.211-3.185$ & 0.033 & 1.279 & $0.631-2.595$ & 0.495 \\
\hline \multicolumn{7}{|l|}{ Positive vs. Negative } \\
\hline Differentiation & 1.912 & $1.035-2.585$ & 0.043 & 1.322 & $0.668-2.614$ & 0.423 \\
\hline \multicolumn{7}{|c|}{ Poor vs. Well/moderate } \\
\hline Clinical stage & 1.969 & $1.104-2.613$ & 0.038 & 1.758 & $0.755-4.093$ & 0.191 \\
\hline$\|B-\| \mid \|$ vs. IA - IAA & & & & & & \\
\hline
\end{tabular}


Inhibitory effects of miR-99b on cell proliferation, migration and invasion of OS cells

To further understand the function of miR-99b in OS progression, HOS and MG63 were applied since they had dramatically low expression of miR-99b. By cell transfection, the expression of miR-99b in both the two cell lines was successfully upregulated by the miR-99b mimic, while was downregulated by the miR-99b inhibitor (all $P<0.01$, Fig. 3a and b). With the use of MTT assay, the cell proliferation of the two cell lines was enhanced by the knockdown of miR-99b, but was inhibited by the overexpression of miR-99b (all $P<0.05$, Fig. 3c and d).

The abilities of migration and invasion were measured in the HOS and MG63 cells after cell transfection by using transwell chambers. As shown in Fig. 4a, the overexpression of miR-99b could suppress, whereas the reduction of miR-99b could promote the cell migration (all $P<0.01$ ). For the invasion assay, we found that the tumor cell invasion was promoted by the knockdown of miR-99b, but was suppressed by the overexpression of miR-99b (all $P<0.05$, Fig. 4b).

\section{Discussion}

Accumulated studies indicate that tumor progression involves the dysregulation of a large number of molecules, of which miRNAs are an important component [18]. Numerous ectopic miRNAs have been identified in various types of tumor and serve critical roles in the pathogenesis of malignancies [19]. For example, the expression of miR-144 was reported to be downregulated in gastric
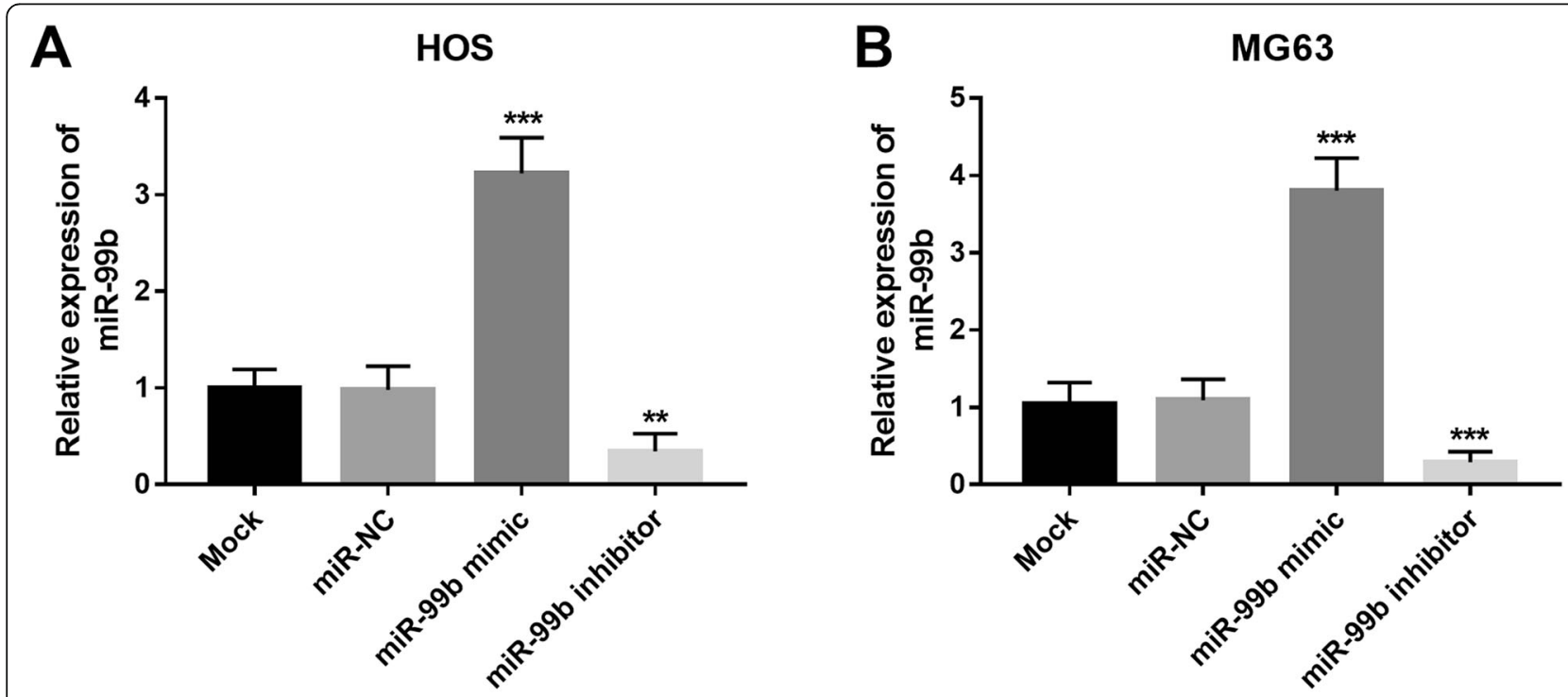

C

HOS

D
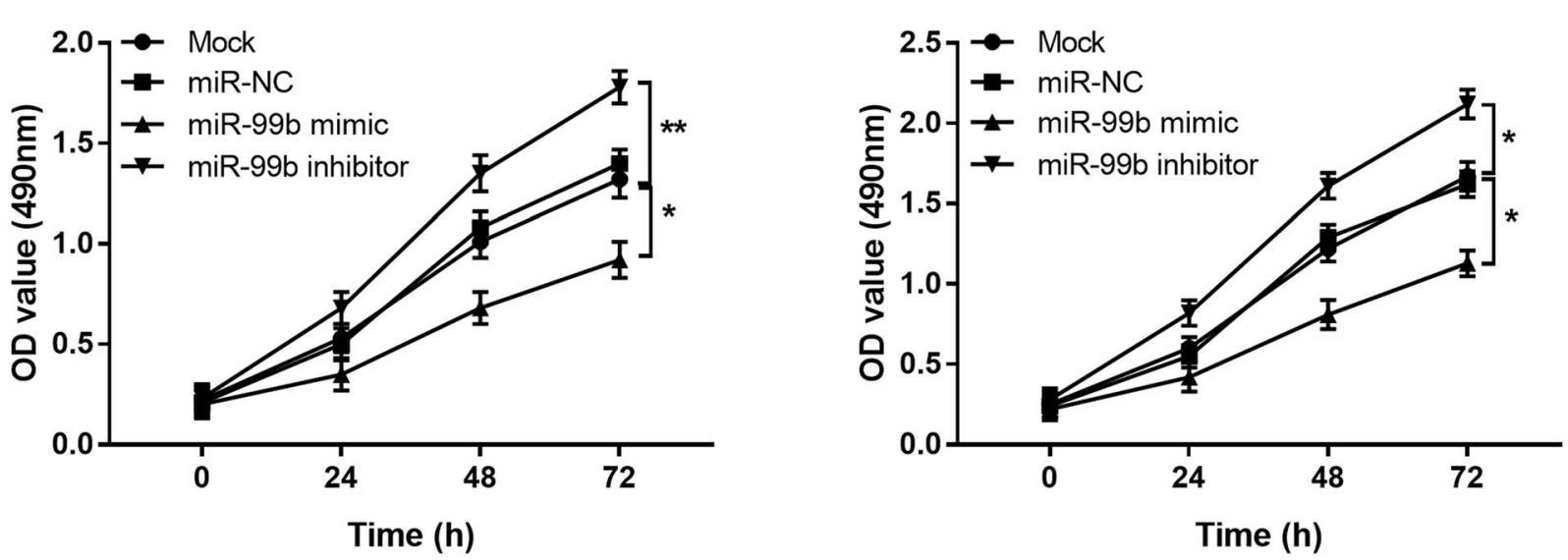

Fig. 3 Effects of miR-99b on cell proliferation of HOS and MG63 cells. a and $\mathbf{b}$ Expression of miR-99b was upregulated by the miR-99b mimic, but was downregulated by the miR-99b inhibitor. $\mathbf{c}$ and $\mathbf{d}$ Tumor cell proliferation was promote by the downregulation of miR-99b, while was inhibited by the upregulation of miR-99b. ${ }^{*} P<0.05,{ }^{*} P<0.01,{ }^{*} P<0.001$ 

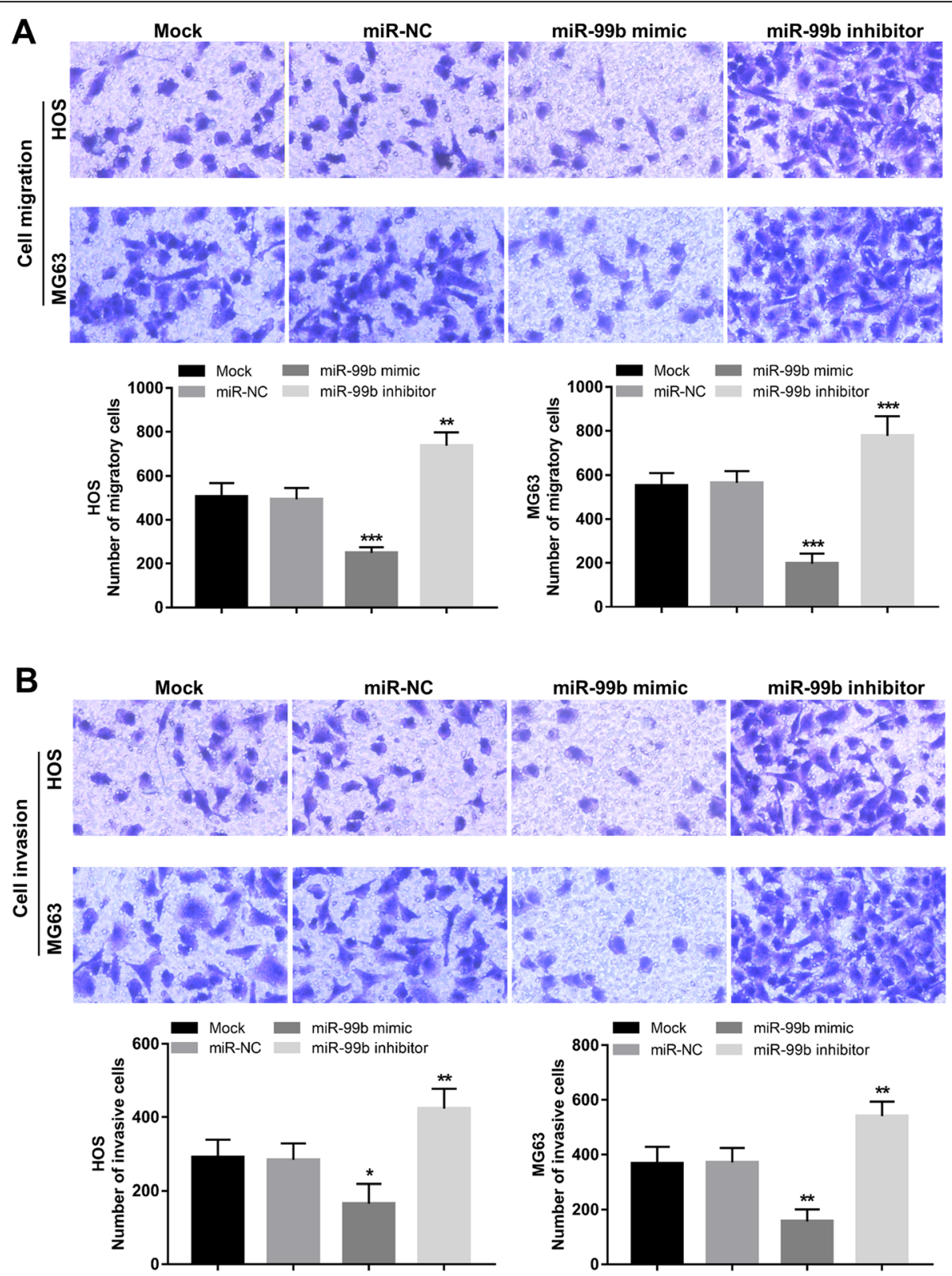

Fig. 4 Effects of miR-99b on cell migration and invasion of HOS and MG63 cells. The overexpression of miR-99b in HOS and MG63 could suppress the cell migration $(\mathbf{a})$ and invasion $(\mathbf{b})$, but the reduction of miR-99b led to the opposite results. ${ }^{*} P<0.05,{ }^{* *} P<0.01,{ }^{* *} P<0.001$

cancer cell lines compared with the normal cells, and the overexpression of miR-144 in tumor cells resulted in inhibited cell proliferation and invasion [20]. In NSCLC tissues, miR-34b was downregulated and associated with the degree of differentiation and pathological stage of the patients, indicating its potential to serve as a biomarker for this malignancy [21]. The aforementioned studies suggest the closely relationship between miRNAs and human malignancies. In OS, there are also some miRNAs with aberrant expression profiles. A study by Chu et al. shown that the expression of miR-136 was reduced in OS tissues and cells, and might be involved in the regulation of tumor cell proliferation, migration and invasion [22]. Another study by Xia et al. found that miR-377 served a tumor suppressor in the tumor progression, which evidenced by the inhibited effects of miR-377 on tumor growth and the enhanced effect on cell apoptosis [23]. Similarly, the decreased miR-139 in OS was also been identified as a suppressor in tumorigenesis, and its suppressive effects on tumor cell proliferation and invasion were exerted by targeting ROCK1 [24]. Taken together, it is important to identify more deregulated miRNAs in the development of OS.

In this study, we found a significantly decreased expression of miR-99b in both the tissue and cell samples of OS 
when compared to the corresponding normal controls. Thus, we considered that the aberrant miR-99b might play a tumor suppressor role in OS. The downregulated expression patterns of miR-99b have been determined in other human cancers, and it has been demonstrated to serve as a tumor suppressor. For instance, miR-99b expression was demonstrated to be reduced in gastric cancer tissues and cell lines, and it could suppress the tumor cell proliferation and cell cycle by targeting IGF-1R [15]. In colorectal carcinoma, the expression of miR-99b was also downregulated in tumor tissues, and tumor cell migration could be inhibited by the overexpression of miR-99b [16]. In our research cohort, we further investigate the relationship between miR-99b expression and the clinical features of the patients, and found that the decreased expression of miR-99b was associated with metastasis and TNM stage. Thus, we considered that the deregulated expression of miR-99b might be related with the development of OS.

Emerging studies highlight the clinical significance of miRNAs in the diagnosis and prognosis of human cancers [25]. The prognostic value of miR-99b has been analyzed in some cancers, such as clear cell renal cell carcinoma [26]. With the recorded survival information, we plotted the survival curves, which indicated that low miR-99b expression was related with shorter survival time. Moreover, the Cox regression data implied that the expression of miR-99b was an independent prognostic indicator. Thus, we considered that the decreased expression of miR-99b could predict poor prognosis of OS.

Considering the reduced expression of miR-99b in OS tissues and cell lines, its functional role in tumor progression was explored after the in vitro regulation of miR-99b. This study found that the OS cell proliferation, migration and invasion were all promoted by the knockdown of miR-99b, while were inhibited by the overexpression of miR-99b, which confirmed that miR-99b served a tumor suppressor in the progression of OS. Although we provided evidence for the inhibiting effects of miR-99b on OS progression, the underlying mechanisms remain unclear. $\mathrm{Li}$ and his colleagues have reported that miR-99b could suppress cervical cancer cell proliferation, invasion, migration and cell cycle by the inhibition of the PI3K/AKT/mTOR signaling pathway [27]. Liu et al. have demonstrated that the non-small cell lung cancer cell proliferation, migration and invasion abilities were downregulated by miR-99b through targeting Frizzled-8 (FZD8) [28]. Furthermore, FZD8 has been reported to serve as an oncogene in OS [29]. Therefore, we suspected that the effects of miR-99b in OS might exerted also through targeting FZD8 or via the PI3K/AKT signaling. Further studies need to be carried out to confirm our hypothesis on the molecular mechanisms. In addition to the limited understanding about the underlying mechanisms, another limitation of this study was the small sample size that might lead to limited accuracy of our results. Thus, future studies are needed with a large research cohort.

In conclusion, all the data in this study demonstrated that the decreased expression of miR-99b is a candidate biomarker in the prognosis of OS. OS tumor cell proliferation, migration and invasion can be suppressed by the overexpression of miR-99b, indicating the potential of miR-99b as a therapeutic target in the treatment of OS.

\section{Abbreviations}

FZD8: Frizzled-8; KM: Kaplan-Meier; miR-99b: MicroRNA-99b; miRNAs: MicroRNAs; OS: Osteosarcoma

\section{Acknowledgements}

Not applicable.

\section{Authors' contributions}

XS and XG analyzed and interpreted the data regarding, performed the examination of cell, and wrote and revised the manuscript. Both authors read and approved the final manuscript.

\section{Funding}

None.

Availability of data and materials

All data generated or analyzed during this study are included in this published article.

\section{Ethics approval and consent to participate}

A signed written informed consent was obtained from each patient and the experimental procedures were all in accordance with the guideline of the Ethics Committee of Qilu Hospital Huantai Branch.

\section{Consent for publication}

Written informed consent for publication was obtained from each participant.

\section{Competing interests}

The authors declare that they have no competing interests.

Received: 17 June 2019 Accepted: 11 September 2019

Published online: 24 October 2019

\section{References}

1. Simpson E, Brown HL. Understanding osteosarcomas. JAAPA. 2018;31(8):15-9.

2. Moreno F, Cacciavillano W, Cipolla M, Coirini M, Streitenberger P, Lopez Marti J, et al. Childhood osteosarcoma: incidence and survival in Argentina. Report from the National Pediatric Cancer Registry, ROHA network 20002013. Pediatr Blood Cancer. 2017;64(10):e26533.

3. Gianferante DM, Mirabello L, Savage SA. Germline and somatic genetics of osteosarcoma - connecting aetiology, biology and therapy. Nat Rev Endocrinol. 2017;13(8):480-91.

4. Heymann MF, Brown HK, Heymann D. Drugs in early clinical development for the treatment of osteosarcoma. Expert Opin Investig Drugs. 2016;25(11):1265-80.

5. Hao H, Chen L, Huang D, Ge J, Qiu Y, Hao L. Meta-analysis of alkaline phosphatase and prognosis for osteosarcoma. Eur J Cancer Care. 2017;26(5):e12536.

6. Zhang X, Guan Z. PET/CT in the diagnosis and prognosis of osteosarcoma. Front Biosci. 2018;23:2157-65.

7. Sun Y, Ye D, Li Y, Chen E, Hao R, Cai Y, et al. CUX2 functions as an oncogene in papillary thyroid cancer. Onco Targets Ther. 2019;12:217-24.

8. Liu Y, Liu T, Jin H, Yin L, Yu H, Bi J. MiR-411 suppresses the development of bladder cancer by regulating ZnT1. Onco Targets Ther. 2018;11:8695-704.

9. Li W, Wang J, Zhang D, Zhang X, Xu J, Zhao L. MicroRNA-98 targets HMGA2 to inhibit the development of retinoblastoma through mediating Wnt/betacatenin pathway. Cancer Biomark. 2019;25(1):79-88.

10. Sun X, Cui S, Fu X, Liu C, Wang Z, Liu Y. MicroRNA-146-5p promotes proliferation, migration and invasion in lung cancer cells by targeting claudin-12. Cancer Biomark. 2019;25(1):89-99. 
11. Hu XH, Dai J, Shang HL, Zhao ZX, Hao YD. miR-1285-3p is a potential prognostic marker in human osteosarcoma and functions as a tumor suppressor by targeting YAP1. Cancer Biomark. 2019;25:1):1-10.

12. Yuan J, Su Z, Gu W, Shen X, Zhao Q, Shi L, et al. MiR-19b and miR-20a suppress apoptosis, promote proliferation and induce tumorigenicity of multiple myeloma cells by targeting PTEN. Cancer Biomark. 2019;24(3):279-89.

13. Cong C, Wang W, Tian J, Gao T, Zheng W, Zhou C. Identification of serum miR-124 as a biomarker for diagnosis and prognosis in osteosarcoma. Cancer Biomark. 2018;21(2):449-54.

14. Wang SN, Luo S, Liu C, Piao Z, Gou W, Wang Y, et al. miR-491 inhibits osteosarcoma lung metastasis and chemoresistance by targeting alphaBcrystallin. Mol Ther. 2017;25(9):2140-9.

15. Wang Z, Zhao Z, Yang Y, Luo M, Zhang M, Wang X, et al. MiR-99b-5p and miR-203a-3p function as tumor suppressors by targeting IGF-1R in gastric cancer. Sci Rep. 2018;8(1):10119.

16. Li W, Chang J, Wang S, Liu X, Peng J, Huang D, et al. miRNA-99b-5p suppresses liver metastasis of colorectal cancer by down-regulating mTOR. Oncotarget. 2015;6(27):24448-62.

17. Gougelet A, Pissaloux D, Besse A, Perez J, Duc A, Dutour A, et al. Micro-RNA profiles in osteosarcoma as a predictive tool for ifosfamide response. Int J Cancer. 2011;129(3):680-90.

18. Huang D, Sun W, Zhou Y, Li P, Chen F, Chen H, et al. Mutations of key driver genes in colorectal cancer progression and metastasis. Cancer Metastasis Rev. 2018;37(1):173-87.

19. O'Bryan S, Dong S, Mathis JM, Alahari SK. The roles of oncogenic miRNAs and their therapeutic importance in breast cancer. Eur J Cancer. 2017;72:1-11.

20. Mushtaq F, Zhang J, Li J. miR-144 suppresses cell proliferation and invasion in gastric cancer through downregulation of activating enhancer-binding protein 4. Oncol Lett. 2019;17(6):5686-92.

21. Yang S, Zhu H, Cheng Q. Correlative analysis of miR-34b and p53 with pathological characteristics of NSCLC. Oncol Lett. 2019;17(6):5558-64.

22. Chu Y, Hu X, Wang G, Wang Z, Wang Y. Downregulation of miR-136 promotes the progression of osteosarcoma and is associated with the prognosis of patients with osteosarcoma. Oncol Lett. 2019;17(6):5210-8.

23. Xia P, Gu R, Zhang W, Shao L, Li F, Wu C, et al. MicroRNA-377 exerts a potent suppressive role in osteosarcoma through the involvement of the histone acetyltransferase 1-mediated Wnt axis. J Cell Physiol. 2019;234:22787.

24. Fan G, He Z, Cao L, Shi X, Wu S, Zhou G. miR-139 inhibits osteosarcoma cell proliferation and invasion by targeting ROCK1. Front Biosci. 2019;24:1167-77.

25. Bertoli G, Cava C, Castiglioni I. MicroRNAs: new biomarkers for diagnosis, prognosis, therapy prediction and therapeutic tools for breast cancer. Theranostics. 2015:5(10):1122-43.

26. Lukamowicz-Rajska M, Mittmann C, Prummer M, Zhong Q, Bedke J, Hennenlotter J, et al. MiR-99b-5p expression and response to tyrosine kinase inhibitor treatment in clear cell renal cell carcinoma patients. Oncotarget. 2016;7(48):78433-47.

27. Li YJ, Wang Y, Wang YY. MicroRNA-99b suppresses human cervical cancer cell activity by inhibiting the PI3K/AKT/mTOR signaling pathway. J Cell Physiol. 2019;234(6):9577-91.

28. Liu R, Chen Y, Shou T, Hu J, Qing C. miRNA-99b-5p targets FZD8 to inhibit non-small cell lung cancer proliferation, migration and invasion. Onco Targets Ther. 2019;12:2615-21.

29. Wang J, Pang W, Zuo Z, Zhang W, He W. MicroRNA-520b suppresses proliferation, migration, and invasion of spinal osteosarcoma cells via downregulation of frizzled-8. Oncol Res. 2017;25(8):1297-304.

\section{Publisher's Note}

Springer Nature remains neutral with regard to jurisdictional claims in published maps and institutional affiliations.

Ready to submit your research? Choose BMC and benefit from:

- fast, convenient online submission

- thorough peer review by experienced researchers in your field

- rapid publication on acceptance

- support for research data, including large and complex data types

- gold Open Access which fosters wider collaboration and increased citations

- maximum visibility for your research: over $100 \mathrm{M}$ website views per year

At BMC, research is always in progress.

Learn more biomedcentral.com/submissions 